\title{
Review Article Epidemiology and Pathogenesis of Providencia alcalifaciens Infections
}

\author{
Mohammad Monir Shah, ${ }^{1,2 \star}$ Erick Odoyo, ${ }^{1}$ and Yoshio Ichinose ${ }^{1,2}$ \\ ${ }^{1}$ Nagasaki University Institute of Tropical Medicine-Kenya Medical Research Institute Project, Nairobi, Kenya; ${ }^{2}$ Centre for Infectious Disease \\ Research in Asia and Africa, Nagasaki University Institute of Tropical Medicine, Nagasaki, Japan
}

\begin{abstract}
Providencia alcalifaciens is a member of the family Enterobacteriaceae that has been commonly implicated as a causative agent of diarrheal infection in humans and animals. Recent outbreaks of $P$. alcalifaciens in both developing and developed countries have raised public health concerns. Several studies have suggested that $P$. alcalifaciens can cause diarrhea by invading the intestinal mucosa, although its pathogenicity has not been well established. Often routine laboratory investigations that seek etiological agents of diarrhea do not actively pursue $P$. alcalifaciens detection. Therefore, routine laboratory diagnosis should be given more attention for better understanding the epidemiology and pathogenicity of $P$. alcalifaciens.
\end{abstract}

\section{INTRODUCTION}

Providencia, a genus of Gram-negative, rod-shaped bacteria, belongs to the family Enterobacteriaceae. Presently, it consists of nine species, ${ }^{1}$ among which Providencia stuartii, Providencia rettgeri, Providencia rustigianii, and Providencia alcalifaciens are the causative agents of different types of infections, including urinary tract infections, wound infections, septicemia, nosocomial infections, and diarrhea. ${ }^{2}$ Several studies have reported that $P$. alcalifaciens is the causative agent of diarrhea in humans and animals, as well as hemorrhagic pneumonia in piglets. ${ }^{3-9}$ Strains of $P$. alcalifaciens isolated from diarrheal patients were able to develop diarrhea in the removal of ileal ties of adult rabbit diarrhea (RITARD) model and showed invasiveness to HEp-2 and other eukaryotic cell lines in vitro, although the pathogenicity of this organism remains poorly established. ${ }^{7,10}$ Previously, three foodborne outbreaks caused by $P$. alcalifaciens were reported in Japan, Turkey, and Kenya, and have attracted considerable attention. ${ }^{11-13}$ This article will present a brief review of the literature regarding the epidemiology and pathogenicity of $P$. alcalifaciens, and justify the importance of routine laboratory diagnosis.

\section{EPIDEMIOLOGY}

Providencia alcalifaciens is commonly found in soil, water, and sewage and has been isolated from a broad range of living organisms such as chickens, dogs, and cows. ${ }^{14}$ The bacterium was first recognized in 1943 by Stuart, who identified it as "anaerogenic paracolon 29911" isolated from diarrheal patients. ${ }^{15}$ Kauffmann labeled these anaerogenic paracolon 29911 strains as Providence group in 1951, based on the report published in Providence, RI by Stuart. ${ }^{2}$ One year later in 1952, Brown found that the anaerogenic paracolon bacilli were responsible for gastroenteritis in children. ${ }^{16}$ In 1955 , Ridge reported a case with the Providence of paracolon bacillus type, similar to the organism previously published by Brown, in a residential nursery. ${ }^{17}$ In the same year, Graber and Lincoln ${ }^{18}$ also found an association between Proteus-

\footnotetext{
*Address correspondence to Mohammad Monir Shah, Centre for Infectious Disease Research in Asia and Africa, Institute of Tropical Medicine, Nagasaki University, 1-12-4 Sakamoto, Nagasaki 8528523, Japan. E-mail: shah@nagasaki-u.ac.jp
}

Providence organisms and infantile diarrhea in Denver. Later, Providencia-associated diarrhea was reported in Nigeria and India. ${ }^{19,20}$ In Bangladesh, Albert and others ${ }^{10}$ were able to demonstrate the association between $P$. alcalifaciens and diarrheal disease in a case-control study by comparing the rates of isolation of the pathogen in children with and those without diarrhea. In 1989, Haynes and Hawkey ${ }^{5}$ implicated $P$. alcalifaciens as a causative agent of traveler's diarrhea. They isolated $P$. alcalifaciens significantly more $(7-8$ times more frequently) among the returning British traveler's with diarrhea than among patients who had diarrhea but had no history of recent travel at the time. Subsequently, this was supported by the findings made by Yoh et al., ${ }^{6}$ in which the pathogen was isolated among traveler's returning to Japan. Furthermore, a cross-sectional study between 2009 and 2013 was able to isolate $3.2 \%$ of $P$. alcalifaciens from the stool samples of diarrheic children in Kenya. ${ }^{21}$ Also, $P$. alcalifaciens is known to cause diarrhea in dogs and hemorrhagic pneumonia in piglets. ${ }^{8,9}$

Transmission of $P$. alcalifaciens occurs primarily through ingestion of contaminated foods, which caused three foodborne outbreaks in Japan, Turkey, and Kenya. ${ }^{11-13}$ In 1996, a large outbreak of foodborne infection caused by $P$. alcalifaciens occurred among children and teachers at two kindergartens and one high school and affected a total of 270 individuals in Fukui, Japan. ${ }^{11}$ Here, no other recognized enteropathogens were detected in fecal samples except $P$. alcalifaciens and the isolates clonality was confirmed by pulsed-field gel electrophoresis. The source of the outbreak was the food eaten at lunch that was prepared and supplied by a single catering facility. In Turkey, $P$. alcalifaciens caused a diarrhea outbreak that affected 27 adults who had consumed potato salad and pork schnitzel during a festive meal. ${ }^{12}$ In Kenya, $P$. alcalifaciens was associated with an outbreak of diarrheal disease in seven primary and four secondary cases where the sole factor was the mashed potatoes eaten by primary cases. ${ }^{13}$ These outbreaks indicate that $P$. alcalifaciens is a potential foodborne pathogen. Thus, it reinforces the need to improve clinical surveillance and laboratory diagnosis.

\section{CLINICAL FEATURES AND DIAGNOSIS}

Diarrheal infection caused by $P$. alcalifaciens usually presents as watery, non-bloody diarrhea, or loose stool, occasionally with abdominal pain, vomiting, fever, and rarely tenesmus. ${ }^{11-13}$ 
The incubation period for $P$. alcalifaciens gastroenteritis ranges from 1 to 4 days, and the frequency of diarrhea ranges from three to 10 episodes per day, although in some instances, up to 15 episodes have been reported. ${ }^{11-13}$ The spectrum of disease severity varies depending on age groups, as observed during the outbreaks in Japan and Kenya. The attack rate was found higher in kindergarten children than in high school students and adults during the outbreak that occurred in Japan. ${ }^{11}$ In addition, in Kenya, severe illness was observed among children compared with the adults who only reported mild diarrhea and required no hospitalization. ${ }^{13}$

Besides diarrheal infections in humans, $P$. alcalifaciens can cause enteritis in puppies, including the clinical signs of dehydration, hypothermia, vocalizing, and blood-tinged diarrhea. $^{8}$ After infection, puppies died within 1-2 days and diffuse superficial mucosal necrosis was observed in the small intestine after necropsies.

Recent studies demonstrated that $P$. alcalifaciens is not only limited to the intestinal infection. Wang and others ${ }^{9}$ described $P$. alcalifaciens-associated hemorrhagic pneumonia in piglets. According to their report, the infected piglets died within 2-4 days after showing clinical symptoms of abdominal skin empurpling, difficulty in breathing, frothy nasal, and oral discharge. The lungs of infected piglets were friable, swollen, and dark brown caused by severe hemorrhage. Interstitial broadening and massive inflammatory cells infiltrated can be seen in histologic sections. However, Vieira et al. ${ }^{22}$ also demonstrated the ability of $P$. alcalifaciens to translocate from the intestinal lumen to extraintestinal sites of the host using a small animal model. It was observed that both invasive and noninvasive $P$. alcalifaciens strains isolated from diarrheal patients were able to resist the human serum bactericidal activity and exhibited the ability to translocate from the gastrointestinal tract to mesenteric lymph nodes, liver, and spleen, which supports the potential role in causing disseminated infection.

Routine laboratory investigations for the identification of etiologic agents of diarrhea do not actively pursue $P$. alcalifaciens because most of the clinical laboratories do not recognize $P$. alcalifaciens as a causative agent of diarrheal disease. Also, it may not be easy to identify or distinguish Providencia species from other non-lactose fermenters such as Salmonella or Shigella species using media such as deoxycholate hydrogen sulfide lactose, MacConkey, and Salmonella-Shigella agar which are often used as selective media for the isolation of bacteria in feces. To circumvent the challenge of isolation and differentiation of Providencia species from other bacteria in culture media, Senior developed P. alcalifaciens medium (PAM) in $1997 .{ }^{23}$ Providencia alcalifaciens medium was found to be a suitable differential medium for $P$. alcalifaciens because it yields red colonies as opposed to yellow or white colonies observed in other Enterobacteriaceae. This is due to the inability of $P$. alcalifaciens to ferment mannitol, xylose, or galactose present in the PAM medium, unlike other fecal bacteria which can ferment one or more of these sugars. Pseudomonas aeruginosa was also found to yield red colonies similar to $P$. alcalifaciens, but they can be easily differentiated using the oxidase test. Providencia aeruginosa is oxidase positive, whereas $P$. alcalifaciens is oxidase negative. Morganella morganii also shows similar growth to $P$. alcalifaciens on PAM medium. Subsequently, Yoh et al. ${ }^{6}$ modified PAM medium by substituting mannitol and galactose with maltose by adding polymyxin-B to establish a selective culture medium known as the polymyxin-mannitolxylitol medium for Providencia (PMXMP). Both the PAM and PMXMP media showed promising results for the isolation and differentiation of Providencia species from other Enterobacteriaceae. In addition, $P$. alcalifaciens can be detected from cultures using commercial API-20E biochemical strip kit (API System; BioMérieux, Marcy-l'Étoile, France) and automated systems such as Vitek-II (BioMérieux). For sensitive and rapid methodologies, polymerase chain reaction-based $16 \mathrm{~S}$ rRNA and dnaJ gene sequences have been used for identification. $^{11,13}$

\section{PATHOGENESIS}

Providencia alcalifaciens-causing diarrheal pathogenicity was first studied by Albert and others ${ }^{10}$ with RITARD model. Studies have shown that the RITARD model has been used to examine diarrheagenic properties in various microorganisms. ${ }^{24-26} \mathrm{How}-$ ever, in this study, $P$. alcalifaciens was isolated from a child and two adults with diarrhea where no other recognized enteric pathogen was detected. All three strains of $P$. alcalifaciens were able to develop diarrhea, whereas some differences were observed in disease-producing abilities. ${ }^{10}$ Two of the three strains developed diarrhea in $80-91 \%$ of experimental animals and colonized the intestinal wall, whereas one strain developed diarrhea in $40 \%$ of the animals and was not recovered from the small intestine of one rabbit during the time of sacrifice. These data indicate the differing disease-producing abilities among various strains of $P$. alcalifaciens. The electron microscopy result of the ileal mucosa indicated two modes of entry into intestinal epithelial cells: one by endocytosis with polymerization of cytoskeletal components and the other by disruption of tight junctions. ${ }^{27}$ On the other hand, none of the strains were able to produce heat-stable enterotoxin in suckling mice, but they develop hindlimb paralysis among rabbits in RITARD model although its relevance with human disease is not clear. ${ }^{10}$

In addition, these three $P$. alcalifaciens isolates showed negative results for heat-labile enterotoxicity in mouse adrenal tumor Y1 cells and cytotoxicity in HeLa cells. They also exhibited adherence to cultured HEp-2 cells and were able to invade cell monolayers with actin condensation. The pattern of this actin condensation was similar to that produced by another invasive organism, Shigella flexneri, but different from enteropathogenic Escherichia coli. Subsequently, a similar type of experiment carried out with 14 additional strains isolated from diarrheal stools confirmed their invasive character and suggested that prior growth at $37^{\circ} \mathrm{C}$ is the most effective for optimal invasiveness. ${ }^{28}$ Khashe et al. ${ }^{29}$ observed that highly invasive $P$. alcalifaciens strains could attach to HEp-2 cell within 2 hours of postinfection, whereas weak or noninvasive strains were found non-adherent, suggesting that the differential attachment may be linked to the key adhesin factors on the cell surface of invasive strains. HeLa cell invasion assay showed that seven of $11 P$. alcalifaciens strains isolated in the São Paulo City, Brazil, have invasive character and actin condensation ability, and no difference was observed when compared with HEp-2 cells. ${ }^{30}$ In addition, Maszewska et al. ${ }^{31}$ demonstrated the invasive phenomenon of $P$. alcalifaciens in Caco- 2 cell lines and compared with HEp- 2 cells. The purpose of using Caco-2 cell lines was to understand the in vivo situation more closely because they can differentiate and form polarized monolayers with a phenotypical resemblance to 
enterocytes of the small intestine. ${ }^{32}$ However, nearly $60 \%$ of $P$. alcalifaciens isolates were invasive into Caco-2 cells, whereas HEp-2 cells showed $95 \%$ invasiveness, suggesting that the level of invasion depends on not only a strain but also on the type of epithelial cells. ${ }^{31}$

Studies have demonstrated that enterobacteria such as Shigella spp., enteroinvasive $E$. coli, and Yersinia enterocolitica harbored plasmid correlates with invasive ability. ${ }^{33-36}$ By contrast, most of the $P$. alcalifaciens studies found no correlation between the presence of plasmids and invasiveness, indicating that not plasmid genes but chromosomal genes are involved in the invasion. 4,31,37 To determine the gene(s) responsible for invasion, TnphoA insertion mutagenesis has been used successfully to screen encoding virulence determinants in a variety of pathogenic bacteria, including $P$. alcalifaciens. ${ }^{38-41}$ Four diarrheal TnphoA mutant $P$. alcalifaciens strains were compared with the parent strain and negligible invasion, and actin condensation in HEp-2 cells was observed. ${ }^{38}$

Beside invasiveness, recently Shima et al. ${ }^{42}$ reported that $P$. alcalifaciens strains could produce the cytolethal distending toxin (CDT) which causes cell elongation, cell distention, and blocks eukaryotic cell proliferation at the G2/M phase, leading to cell death. Since 1987, CDT toxin has been reported in various Gram-negative bacteria such as E. coli, Haemophilus ducreyi, Helicobacter, Campylobacter, and Shigella spp., which are composed of three polypeptides, namely, $\mathrm{CdtA}, \mathrm{CdtB}$, and CdtC, that form a complex structure required for the toxin activity. ${ }^{43}$ Some studies have documented the isolation and characterization of CDT-producing bacteria from patients with diarrhea. ${ }^{44-47}$ However, CDT of $P$. alcalifaciens showed some homology with the CDT of Shigella boydii, suggesting that the CDT gene cluster of $P$. alcalifaciens might be horizontally transferable. This suggests that $P$. alcalifaciens may be similar in terms of pathogenicity with $S$. boydii and this could explain, in part, why $P$. alcalifaciens may not be overly invasive. It is however important to note that only some of the $P$. alcalifaciens studied were shown to harbor the CDT genes. Moreover, further characterization of the identified $P$. alcalifaciens CDT would be required to confirm it as a virulence factor.

Another study suggested that $P$. alcalifaciens uses manganese superoxide dismutase (Mn-SOD) for intra-phagocytic survival, whereas sodA-deleted mutant strain showed lower virulence properties than wild type. ${ }^{48}$ Also, Mn-SOD protects $P$. alcalifaciens from murine macrophage cells. This result provides evidence that Mn-SOD of $P$. alcalifaciens is involved in invasive activity, resulting in intracellular survival. Recently, it has been shown that lipopolysaccharides were a potent inducer of epithelial barrier dysfunction and endothelial cytotoxicity during $P$. alcalifaciens infection. ${ }^{49}$

\section{CONCLUSION}

In recent years, several foodborne outbreaks caused by $P$. alcalifaciens have confirmed the increasing incidence of diarrhea due to $P$. alcalifaciens in the world, regardless of the socioeconomic strata. And this incidence is probably higher than that reported because it is mostly implicated as a self-limiting diarrheagenic pathogen and usually causes moderate morbidity. In addition, most studies conducted within the tropics do not properly consider $P$. alcalifaciens as a possible etiologic agent of diarrhea, and thus do not actively detect the organisms as well as focus on epidemiological aspects. Invasiveness remains the most established mode of pathogenesis in $P$. alcalifaciens, and future research on this matter is needed. The incidence of $P$. alcalifaciens-causing gastroenteritis can be reduced by good hygiene and food preparation. Detection of the pathogen routinely at clinical laboratories is strongly recommended; this along with integrated surveillance programs can be effective for the control of $P$. alcalifaciens infections.

Received May 2, 2018. Accepted for publication February 20, 2019.

Published online June 17, 2019.

Acknowledgment: We acknowledge the contribution of Betty Muriithi of the NUITM-KEMRI project in this manuscript.

Authors' addresses: Mohammad Monir Shah and Yoshio Ichinose, Nagasaki University Institute of Tropical Medicine-Kenya Medical Research Institute Project, Nairobi, Kenya and Centre for Infectious Disease Research in Asia and Africa, Nagasaki University Institute of Tropical Medicine, Nagasaki, Japan, E-mails: shah@nagasaki-u.ac.jp and ichinose@nagasaki-u.ac.jp. Erick Odoyo, Nagasaki University Institute of Tropical Medicine-Kenya Medical Research Institute Project, Nairobi, Kenya, E-mail: e.odoyo@gmail.com.

\section{REFERENCES}

1. DI H, Liang S, Li Q, Shi L, Shima A, Meng H, Yan H, Yamasaki S, 2018. Providencia in retail meats from Guangzhou, China and Osaka, Japan: prevalence, antimicrobialresistance and characterization of classes 1, 2 and 3 integrons. J Vet Med Sci 80: 829-835.

2. O'Hara CM, Brenner FW, Miller JM, 2000. Classification, identification, and clinical significance of Proteus, Providencia, and Morganella. Clin Microbiol Rev 13: 534-546.

3. Albert MJ, Faruque ASG, Mahalanabis D, 1998. Association of Providencia alcalifaciens with diarrhea in children. J Clin Microbiol 36: 1433-1435.

4. Guth BEC, Perrella E, 1996. Prevalence of invasive ability and other virulence-associated characteristics in Providencia alcalifaciens strains isolated in São Paulo, Brazil. J Med Microbiol 45: 459-462.

5. Haynes J, Hawkey PM, 1989. Providencia alcalifaciens and travellers' diarrhoea. BMJ 299: 94-95.

6. Yoh M, Matsuyama J, Ohnishi M, Takagi K, Miyagi H, Mori K, Park KS, Ono T, Honda T, 2005. Importance of Providencia species as a major cause of traveler's diarrhoea. JMed Microbio/54: 1077-1082.

7. Janda JM, Abbott SL, Woodward D, Khashe S, 1998. Invasion of HEp-2 and other eukaryotic cell lines by Providencia: further evidence supporting the role of Providencia alcalifaciens in bacterial gastroenteritis. Curr Microbiol 37: 159-165.

8. Möhr AJ, van der Lugt JJ, Josling D, Picard J, van der Merwe LL, 2002. Primary bacterial enteritis caused by Providencia alcalifaciens in three dogs. Vet Rec 150: 52-53.

9. Wang X, Wang J, Hao H, Qiu L, Liu H, Chen S, Dang R, Yang Z, 2014. Pathogenic Providencia alcalifaciens strain that causes fatal hemorrhagic pneumonia in piglets. Curr Microbiol 68: 278-284.

10. Albert MJ, Alam K, Ansaruzzaman M, Islam MM, Rahman AS, Haider K, Bhuiyan NA, Nahar S, Ryan N, Montanaro J, 1992. Pathogenesis of Providencia alcalifaciens-induced diarrhea. Infect Immun 60: 5017-5024.

11. Murata T, lida T, Shiomi Y, Tagomori K, Akeda Y, Yanagihara I, Mushiake S, Ishiguro F, Honda T, 2001. A large outbreak of foodborne infection attributed to Providencia alcalifaciens. $J$ Infect Dis 184: 1050-1055.

12. Chlibek R, Jirous J, Beran J, 2002. Diarrhea outbreak among Czech Army Field Hospital personnel caused by Providencia alcalifaciens. J Travel Med 9: 151-152.

13. Shah MM, Odoyo E, Larson PS, Apondi E, Kathiiko C, Miringu G, Nakashima M, Ichinose Y, 2015. First report of a foodborne Providencia alcalifaciens outbreak in Kenya. Am J Trop Med Hyg 93: 497-500.

14. Genthe NA, Thoden JB, Benning MM, Holden HM, 2015. Molecular structure of an $\mathrm{N}$-formyltransferase from Providencia alcalifaciens O30. Protein Sci 24: 976-986. 
15. Stuart CA, Wheeler KM, Rustigian R, Zimmerman A, 1943. Biochemical and antigenic relationships of the paracolon bacteria. J Bacteriol 45: 101-119.

16. Brown GW, 1952. Anaerogenic paracolon bacilli associated with gastro-enteritis in children. Med J Aust 2: 658-664.

17. Ridge LE, Thomas ME, 1955. Infection with the Providence type of paracolon bacillus in a residential nursery. $J$ Pathol Bacteriol 69: 334-337.

18. Graber CD, Lincoln AF, 1955. Infantile diarrhea in the Denver area: significance of Proteus-Providence organisms. Pediatrics 16: 585-589.

19. Sen $R, 1962$. Isolation of strains of the providence group from cases with diarrheoa in Ibadan, Nigeria, West Africa. Indian $\mathrm{J}$ Med Res 50: 622-626.

20. Bhat $P$, Myers RM, Feldman RA, 1971. Providence group of organisms in the aetiology of juvenile diarrhoea. Indian J Med Res 59: 1010-1018.

21. Shah M, Kathiiko C, Wada A, Odoyo E, Bundi M, Miringu G, Guyo $S$, Karama M, Ichinose Y, 2016. Prevalence, seasonal variation, and antibiotic resistance pattern of enteric bacterial pathogens among hospitalized diarrheic children in suburban regions of central Kenya. Trop Med Health 44: 39.

22. Vieira ABR, Koh IHJ, Guth BEC, 2003. Providencia alcalifaciens strains translocate from the gastrointestinal tract and are resistant to lytic activity of serum complement. J Med Microbiol 52: 633-636.

23. Senior BW, 1997. Media for the detection and recognition of the enteropathogen Providencia alcalifaciens in faeces. $J$ Med Microbiol 46: 524-527.

24. Albert MJ, Alam K, Islam M, Montanaro J, Rahaman AS, Haider K, Hossain MA, Kibriya AK, Tzipori S, 1991. Hafnia alvei, a probable cause of diarrhea in humans. Infect Immun 59: 1507-1513.

25. Pazzaglia G, Sack RB, Bourgeois AL, Froehlich J, Eckstein J, 1990. Diarrhea and intestinal invasiveness of Aeromonas strains in the removable intestinal tie rabbit model. Infect Immun 58: 1924-1931.

26. Spira WM, Sack RB, Froehlich JL, 1981. Simple adult rabbit model for Vibrio cholerae and enterotoxigenic Eschenichia coli diarrhea. Infect Immun 32: 739-747.

27. Mathan MM, Mathan VI, Albert MJ, 1993. Electron microscopic study of the attachment and penetration of rabbit intestinal epithelium by Providencia alcalifaciens. J Pathol 171: 67-71.

28. Albert MJ, Ansaruzzaman M, Bhuiyan NA, Neogi PKB, Faruque ASG, 1995. Characteristics of invasion of HEp-2 cells by Providencia alcalifaciens. J Med Microbiol 42: 186-190.

29. Khashe S, Scales DJ, Abbott SL, Janda JM, 2001. Non-invasive Providencia alcalifaciens strains fail to attach to HEp-2 cells. Curr Microbiol 43: 414-417.

30. Magalhaes V, Leal NC, Melo VM, Sobreira M, Magalhaes M, 1996. Invasion of HeLa cells by Providencia alcalifaciens presumably is plasmid-encoded. Mem Inst Oswaldo Cruz 91: 767-768.

31. Maszewska A, Torzewska A, Staczek P, Rozalski A, 2010. Enterocyte-like Caco-2 cells as a model for in vitro studies of diarrhoeagenic Providencia alcalifaciens invasion. Microb Pathog 49: 285-293.

32. Hidalgo IJ, Raub TJ, Borchardt RT, 1989. Characterization of the human colon carcinoma cell line (Caco-2) as a model system for intestinal epithelial permeability. Gastroenterology 96: 736-749.

33. Sansonetti PJ, Kopecko DJ, Formal SB, 1982. Involvement of a plasmid in the invasive ability of Shigella flexneri. Infect Immun 35: 852-860.
34. Silva RM, Toledo MRF, Trabulsi LR, 1982. Correlation of invasiveness with plasmid in enteroinvasive strains of Escherichia coli. J Inject Dis 146: 706.

35. Harris JR, Wachsmuth IK, Davis BR, Cohen ML, 1982. Highmolecular-weight plasmid correlates with Escherichia coli enteroinvasiveness. Infect Immun 37: 1295-1298.

36. Zink DL, Feeley JC, Wells JG, Vanderzant C, Vickery JC, Roof WD, O'Donovan GA, 1980. Plasmid-mediated tissue invasiveness in Yersinia enterocolitica. Nature 283: 224-226.

37. Sobreira M, Leal NC, Magalhães M, Guth BE, Almeida AM, 2001. Molecular analysis of clinical isolates of Providencia alcalifaciens. J Med Microbiol 50: 29-34.

38. Rahman M, Monira S, Nahar S, Ansaruzzaman M, Alam K, Alam M, Albert MJ, 2002. TnphoA mutants of Providencia alcalifaciens with altered invasiveness of HEp-2 cells. J Med Microbiol 51: 682-686.

39. Taylor RK, Miller VL, Furlong DB, Mekalanos JJ, 1987. Use of phoA gene fusions to identify a pilus colonization factor coordinately regulated with cholera toxin. Proc Natl Acad Sci USA 84: 2833-2837.

40. Donnenberg MS, Calderwood SB, Donohue-Rolfe A, Keusch GT, Kaper JB, 1990. Construction and analysis of TnphoA mutants of enteropathogenic Escherichia coli unable to invade Hep-2 cells. Infect Immun 58: 1565-1571.

41. Allaoui A, Sansonetti PJ, Parsot C, 1992. MxiJ, a lipoprotein involved in secretion of Shigella Ipa invasins, is homologous to YscJ, a secretion factor of the Yersinia Yop proteins. J Bacteriol 174: 7661-7669.

42. Shima A, Hinenoya A, Asakura M, Sugimoto N, Tsukamoto T, Ito H, Nagita A, Faruque SM, Yamasaki S, 2012. Molecular characterizations of cytolethal distending toxin produced by Providencia alcalifaciens strains isolated from patients with diarrhea. Infect Immun 80: 1323-1332.

43. Yamasaki S, Asakura M, Tsukamoto T, Faruque SM, Deb R, Ramamurthy T, 2008. Cytolethal distending toxin (CDT): genetic diversity, structure and role in diarrheal disease. Toxin Rev 25: $61-88$

44. Albert MJ, Faruque SM, Faruque AS, Bettelheim KA, Neogi PK, Bhuiyan NA, Kaper JB, 1996. Controlled study of cytolethal distending toxin-producing Escherichia coli infections in Bangladeshi children. J Clin Microbiol 34: 717-719.

45. Pandey $\mathrm{M}$ et al., 2003. Association of cytolethal distending toxin locus cdtB with enteropathogenic Escherichia coli isolated from patients with acute diarrhea in Calcutta, India. J Clin Microbiol 41: 5277-5281.

46. Bouzari S, Oloomi M, Oswald E, 2005. Detection of the cytolethal distending toxin locus $c d t B$ among diarrheagenic Escherichia coli isolates from humans in Iran. Res Microbiol 156: 137-144.

47. Hinenoya A, Naigita A, Ninomiya K, Asakura M, Shima K, Seto K, Tsukamoto T, Ramamurthy T, Faruque SM, Yamasaki S, 2009. Prevalence and characteristics of cytolethal distending toxinproducing Escherichia coli from children with diarrhea in Japan. Microbiol Immunol 53: 206-215.

48. Chen X, Kodama T, lida T, Honda T, 2007. Demonstration and characterization of manganese superoxide dismutase of Providencia alcalifaciens. Microbiol Immunol 51: 951-961.

49. Asakura $\mathrm{H}$, Momose $\mathrm{Y}$, Ryu $\mathrm{CH}$, Kasuga $\mathrm{F}$, Yamamoto $\mathrm{S}$, Kumagai S, Igimi S, 2013. Providencia alcalifaciens causes barrier dysfunction and apoptosis in tissue cell culture: potent role of lipopolysaccharides on diarrheagenicity. Food Addit Contam Part A Chem Anal Control Expo Risk Assess 30: 1459-1466. 